\title{
Demographic and Clinical Differences of Aggressive and Non-Aggressive Suicide Attempts in The Emergency Department in the Eastern Region of Turkey
}

\author{
Atif Bayramoglu ${ }^{1, *}$; Murat Saritemur ${ }^{1} ;$ Sultan Tuna Akgol Gur ${ }^{2}$; Mucahit Emet $^{1}$ \\ ${ }^{1}$ Department of Emergency Medicine, Medicine Faculty, Ataturk University, Erzurum, Turkey \\ ${ }^{2}$ Department of Emergency Medicine, Regional Training and Research Hospital, Erzurum, Turkey \\ ${ }^{*}$ Corresponding Author: Atif Bayramoglu, Department of Emergency Medicine, Medicine Faculty, Ataturk University, Erzurum, Turkey. Tel: +90-4423448407, Fax: +90-4422361301, \\ E-mail:atifbay@gmail.com
}

Received: October 18, 2014; Revised: November 27, 2014; Accepted: December 13, 2014

\begin{abstract}
Background: Worldwide, suicide ranks among the three leading causes of death among those aged 15-44 years. An estimated 800000 people die by committing suicide annually.

Objectives: To better understand the association between aggressive suicidal attempt and non-aggressive suicidal attempt in patients with suicide attempt in the emergency department.

Patients and Methods: A cross-sectional observational study was conducted on suicide attempters of eastern region of Turkey between May 2008 and January 2012. Information of all adult suicide attempts was collected prospectively on a form. Suicide attempts were grouped as aggressive and non-aggressive attempts on a specially designed data-collection form. The aggressive suicide attempts contained violent suicide methods such as firearm, hanging, jumping, car exhaust or drowning.

Results: A total of 533 patients were included. Sixty-four of these patients admitted to ED with aggressive suicide attempt (12\%). Nonaggressive suicide attempts were consulted to psychiatry more compared to aggressive ones (\%73.6, $\mathrm{n}=345 \mathrm{vs}$. \%32.8, $\mathrm{n}=21, \mathrm{P}<0.0001$ ). Agitation in ED and being male increased aggressive suicide attempt risk by 3.5 (\%95 CI: 1.6-7.6) and 3.2 times (\%95 CI: 1.8-5.5), agitated patients in ED group and male group respectively. Patients with aggressive suicide attempt were statistically more frequent among these patients; patients with agitation, those hospitalized in intensive care or surgical services and those whose length of stay in the emergency department was less than one day $(\mathrm{P}<0.05$ for all). Patients with non-aggressive suicide attempt were statistically more frequent in these patients; patients complained of nausea, vomiting, stomach pain, fatigue, those with confusion, those tending to sleep, those hospitalized in internal services or emergency ward and finally those whose length of stay in the emergency department was more than one day $(\mathrm{P}<$ 0.05 for all).

Conclusions: Patients with aggressive suicide attempt who have high risk of dying should be recognized and requested psychiatric consultation even if not in the emergency department.
\end{abstract}

Keywords:Violence; Suicide, Attempt; Suicide; Demography

\section{Background}

Worldwide, suicide ranks among the three leading causes of death in those aged 15-44 years. An estimated 800000 people die by committing suicide annually. This represents one death every 40 seconds (1). While hanging was the predominant method of suicide in most countries, especially in Eastern Europe (i.e. Estonia, Latvia, Lithuania, Poland and Romania), firearm suicide is the most common method in the United States, Argentina, Switzerland and Uruguay. Jumping from a height plays an important role in Hong Kong SAR, Luxembourg and Malta. Violent and highly lethal methods such as firearm suicide and hanging are more frequent among men, whereas women often choose poisoning or drowning, which are less violent and lethal (2). Regarding the methods for suicidal attempt in patients admitted to our hospital, drugs, gunshot wounds, hanging, jumping from high places, self-mutilation by cutting-piercing tools, exposure to toxic substances and other methods can be mentioned.

\section{Objectives}

In this study, we aimed to evaluate demographic properties and clinical features of patients admitted to the emergency service of our hospital for suicidal attempt regarding their method of choice.

\section{Patients and Methods}

All aspects of the study protocol were authorized by the medical ethics committee and the local health authorities prior to initiation of this study (date: 21.04.2009; No: B.30.2.ATA.0.01.00/132).

Copyright (C) 2015, Iranian Red Crescent Medical Journal. This is an open-access article distributed under the terms of the Creative Commons Attribution-NonCommercial 4.0 International License (http://creativecommons.org/licenses/by-nc/4.0/) which permits copy and redistribute the material just in noncommercial usages, provided the original work is properly cited. 


\subsection{Study Design}

This was a cross-sectional observational study on all adult suicide attempts brought to Ataturk University Medical Faculty hospital, which is a governmental referral hospital located in Erzurum in the eastern region of Turkey between May 2008 and January 2012 (45 months). The population of the city Centre of Erzurum is 383000 , and the population of province is 769000 . Although our hospital primarily provides health services for individuals who live in the city center, it provides services for individuals from the counties and villages of Erzurum and 11 neighboring cities, comprising a total of four million individuals (3). The hospital provides healthcare and emergency treatment for all illnesses and accidents. Our hospital did not have a self-harm team at the time of study. Information was collected prospectively on a specially designed data collection form. It included age, gender, address, stated date and time of overdose, time of admission, type of suicide attempt, time of discharge, type of ingested drug, whether other drugs had been coingested, chief complaints, any history of psychiatric illnesses in patient or his or her first-degree relatives, previous suicidal attempts by patient or his or her first-degree relatives, and previous psychiatry polyclinic admission in the last six months.

\subsection{Inclusion and Exclusion Criteria}

Patients 14 years of age and older presented to the ED for care due to suicide attempts by oral ingestion, or suicidal self-injury, were included $(\mathrm{n}=587)$. Patients younger than 14 years brought to the pediatric ED for care due to suicide attempts by oral ingestion, drug intoxication, and non-suicidal accidents and self-injuries were excluded from the study. Patients who engaged in mutilation without suicidal intent and repetitive superficial bodily harm without suicidal intent were not included. Patients who were unable to fill out the study form at the time of arrival at the ED, those for whom data could not be obtained from relatives ( $n=17)$, and those who left the ED without filling out the study form were excluded $(n=22)$. Besides, patients who did not consent to participate in the study were excluded $(n=15)$. Finally, 533 patients were enrolled.

\subsection{Definitions}

Aggression defined as feelings of anger or antipathy resulting in hostile or violent behavior. According to Traskman et al. suicide attempt method was defined as violent (e.g. firearm, hanging, jumping, car exhaust, drowning) or nonviolent (drug overdose or superficial phlebotomy) (4). A suicide attempt is defined as an act with a non-fatal outcome in which an individual deliberately ingests a substance in excess of prescribed or generally recognized therapeutic dosage (5). Suicide attempts are divided into two groups, namely those including and those not including violence. Violent methods (aggressive suicides) include hanging, jumping off a tall building, self-burning, driving a car off a cliff and using sharp objects or firearms to harm oneself. Nonviolent methods (non-aggressive suicides) include attempts made through drug ingestion and the use of gas (6). Table 1 presents patients' suicide attempt methods. Co-ingestion of drugs from different classes was accepted as multidrug ingestion. The co-ingestion of alcohol or tetrahydrocannabinol was not accepted as multidrug ingestion.

\subsection{Data Analysis}

Informed consent was obtained from each patient. SPSS.19 (Statistical Package for Social Sciences) for Mac (SPSS Inc., Chicago, IL, USA) program was used to analyze data. The mean values were shown together with standard deviations. Statistical analysis of the categorical variables was performed using chi-square test or Fisher's exact test, and the analysis of numerical variables was performed using t-test. The variables were tested for normality using the Kolmogorov-Smirnov test. The data were divided into groups, and regression analysis was performed. P value $<$ 0.05 was accepted as statistically significant.

\section{Results}

\subsection{Baseline Sociodemographic and Clinical Char- acteristics}

Table 1. Evaluation of Sociocultural Characteristics of Patients for Their Method of Choice for Suicide ${ }^{a}$

\begin{tabular}{|c|c|c|c|}
\hline $\begin{array}{l}\text { Sociocultural } \\
\text { characteristics }\end{array}$ & Aggressive & $\begin{array}{c}\text { Non- } \\
\text { Aggressive }\end{array}$ & PValue \\
\hline Age, $y$ & $24,26.4 \pm 11.9$ & $22,25.5 \pm 9.7$ & 0.516 \\
\hline \multicolumn{4}{|l|}{ Gender } \\
\hline Female & $29(45.3)$ & $327(69.7)$ & $<0.0001$ \\
\hline $\begin{array}{l}\text { Erzurum prov- } \\
\text { ince }\end{array}$ & $45(70.3)$ & $321(68.4)$ & 0.762 \\
\hline Marital Status & & & 0.409 \\
\hline Married & $28(43.8)$ & $180(38.4)$ & \\
\hline Single & $36(56.3)$ & $289(61.6)$ & \\
\hline Occupation & & & 0.066 \\
\hline Working & $11(17.2)$ & $92(19.6)$ & \\
\hline Non-occupied & $35(54.7)$ & $247(52.7)$ & \\
\hline Student & $12(18.8)$ & $116(24.7)$ & \\
\hline Unknown & $6(9.4)$ & $14(3.0)$ & \\
\hline $\begin{array}{l}\text { Rural or Urban } \\
\text { Inhabitance }\end{array}$ & & & 0.405 \\
\hline Village & $4(6.3)$ & $39(8.3)$ & \\
\hline District & $14(21.9)$ & $73(15.6)$ & \\
\hline City Center & $46(71.9)$ & $357(76.1)$ & \\
\hline
\end{tabular}

\footnotetext{
a Data are presented as No. (\%) or Mean \pm SD.
} 
Of 533 patients enrolled in the study, $66.8 \%(n=356)$ were female, and patients' mean and median age were $25.7 \pm$ 9.9 and 23 years ( $\min 14, \max 88$ ). The female to male ratio was 2:1. The suicide method and number of patients are shown in Table 1 . When sociocultural characteristics were evaluated for suicide attempt methods, non-aggressive suicide attempts were more common in females and aggressive attempts in males (Table 2). A psychiatric consultation was requested for 366 (68.3\%) patients in the ED. There was a history of psychiatric clinic admission within the previous six months in 121 (22.7\%) patients. Of 533 patients, 396 (74.3\%) were hospitalized, 82 (15.4\%) were discharged from the ED and 55 (10.3\%) left the hospital. Moreover, $6.2 \%(n=4)$ of aggressive suicide attempters and $10.9 \%(n=51)$ of non-aggressive suicide attempters did not agree to the evaluation $(\mathrm{P}=0.254)$. While drugrelated symptoms such as nausea, vomiting, abdominal pain, somnolence and fatigue were more frequent in non-aggressive suicides, loss of consciousness and agitation were more common in aggressive suicides (Table 3). Regarding the ward that patients were hospitalized, aggressive suiciders were admitted to the emergency service significantly at a higher rate than non-aggressive ones (76.5\% vs. 9.4\% P $<0.0001$ ), whereas aggressive suiciders were more frequently admitted to the surgical services (67.2 \% vs. 13.6\%, P < 0.0001). While aggressive suiciders stayed under observation for less than one day in our emergency service, non-aggressive suiciders were observed for longer periods (Table 3). When psychiatric characteristics were analyzed for suicide methods, the rate of psychiatry consultation in the emergency service was found to be significantly lower in patients with aggressive attempts than those with non-aggressive at- tempts (32.8\% vs. 73.6\%; P < 0.0001) (Table 4). This might probably due to the inability to consult with psychiatry, because of poor general medical condition of patients with aggressive attempts. When clinical and sociodemographic characteristics of patients who committed aggressive suicide were compared with those who did not commit aggressive suicide by enter method and logistic regression analysis, the probability of being male increased by 2.8 fold (95\% CI:1.6-5.0). The rate of psychiatry consultation in the emergency service increased by 4.8 fold (95\% CI: 2.7-8.8) in patients with non-aggressive suicidal attempts and they were found to be manifesting agitation 5.2 fold more than those with aggressive suicidal attempts (95\% CI: 2.2-12.2) (Table 5).

Table 2. Patients According to Suicide Attempt Methods a

\begin{tabular}{|lcc}
\hline Type of suicide & Results & CI \%95 \\
\hline Aggressive attempt & $72(13.5)$ & $10.9-16.7$ \\
\hline Hanging & $9(1.7)$ & $0.9-3.2$ \\
\hline Cutting & $23(4.3)$ & $2.9-6.4$ \\
\hline Firearm & $8(1.5)$ & $0.7-2.9$ \\
\hline Drug overdose + Cutting & $8(1.5)$ & $0.7-2.9$ \\
\hline Gas inhalation + Cutting & $2(0.4)$ & $0.1-1.4$ \\
\hline Jumping & $18(3.4)$ & $2.2-5.3$ \\
\hline Drinking Corrosives & $4(0.8)$ & $0.3-1.9$ \\
\hline Non-Aggressive attempt & $461(86.5)$ & $83.3-89.1$ \\
\hline Drug overdose & $459(86.1)$ & $82.9-88.8$ \\
\hline Gas inhalation ${ }^{\mathrm{b}}$ & $2(0.4)$ & $0.1-1.4$ \\
\hline Total & 533 & 100 \\
\hline
\end{tabular}

a Data are presented as No. (\%).

${ }^{\mathrm{b}}$ Natural gas or liquefied petroleum gas.

Table 3. Evaluation of Clinical Features of Patients for Suicide Methods ${ }^{\text {a }}$

\begin{tabular}{|c|c|c|c|}
\hline Clinical feature & Aggressive & Non-Aggressive & P Value \\
\hline Nausea, yes/no & $11(17.2)$ & $265(56.5)$ & $<0.0001$ \\
\hline Vomiting, yes/no & $2(3.1)$ & $172(36.7)$ & $<0.0001$ \\
\hline Abdominal Pain, yes/no & $7(10.9)$ & $125(26.7)$ & 0.006 \\
\hline Somnolence, yes/no & $4(6.3)$ & $185(39.4)$ & $<0.0001$ \\
\hline Loss of consciousness, yes/no & $12(18.8)$ & $44(9.4)$ & 0.022 \\
\hline Headache, yes/no & $5(7.8)$ & $38(8.1)$ & 0.929 \\
\hline Vertigo, yes/no & $3(4.7)$ & $51(10.9)$ & 0.124 \\
\hline Agitation, yes/no & $12(18.8)$ & $31(6.6)$ & 0.001 \\
\hline Urine retention, yes/no & $0(0.0)$ & $18(3.8)$ & 0.111 \\
\hline Fatigue, yes/no & $3(4.7)$ & $63(13.4)$ & 0.046 \\
\hline Prognosis discharge & $16(25.0)$ & $66(14.1)$ & 0.054 \\
\hline Admittance & $44(68.8)$ & $352(75.1)$ & \\
\hline Leaving the hospital & $4(6.3)$ & $51(10.9)$ & \\
\hline Admitted to Emergency Service Ward & $6(9.4)$ & $359(76.5)$ & $<0.0001$ \\
\hline Internal medicine & $7(10.9)$ & $26(5.5)$ & \\
\hline Intensive care & $8(12.5)$ & $20(4.3)$ & \\
\hline Surgical services & $43(67.2)$ & $64(13.6)$ & \\
\hline Hospitalization Period less than one day & $64(100.0)$ & $375(80.0)$ & $<0.0001$ \\
\hline More than one day & $0(0.0)$ & $94(20.0)$ & \\
\hline
\end{tabular}

${ }^{\mathrm{a}}$ Data are presented as No. (\%). 
Bayramoglu A et al.

\begin{tabular}{|c|c|c|c|}
\hline Psychiatric Characteristics & Aggressive & Non-Aggressive & PValue \\
\hline Consultation with psychiatry, yes/no & $21(32.8)$ & $345(73.6)$ & $<0.0001$ \\
\hline $\begin{array}{l}\text { Admittance to psychiatry in the last } \\
\text { six months, yes/no }\end{array}$ & $9(14.1)$ & $112(23.9)$ & 0.079 \\
\hline $\begin{array}{l}\text { Family history of psychiatric disease, } \\
\text { yes/no }\end{array}$ & $5(7.8)$ & $61(13.0)$ & 0.237 \\
\hline Previous psychiatric diagnosis, yes/no & $15(23.4)$ & $124(26.4)$ & 0.608 \\
\hline Family history of suicide, yes/no & $6(9.4)$ & $26(5.5)$ & 0.226 \\
\hline Previous suicide attempt, yes/no & $12(18.8)$ & $69(14.7)$ & 0.399 \\
\hline
\end{tabular}

${ }^{\mathrm{a}}$ Data are presented as No.(\%).

Table 5. Comparison of Clinical and Sociodemographic Characteristics of Patients Who Committed Aggressive Suicide With Those Who Did Not Commit Aggressive Suicide by Logistic Regression Analysis

\begin{tabular}{lcccc}
\hline Clinical and Sociodemographic Char- & P & OR & \multicolumn{2}{c}{ 95\% C.I. } \\
\cline { 2 - 5 } acteristics of Patients & Lower & Upper & Lower & Upper \\
\hline Gender & 0.001 & 2.800 & 1.566 & 5.006 \\
\hline Agitation, yes/no & $<0.0001$ & 5.152 & 2.175 & 12.205 \\
$\begin{array}{l}\text { Admittance to psychiatry in the last } \\
\text { six months, Yes/No }\end{array}$ & 0.278 & 0.640 & 0.286 & 1.433 \\
Psychiatry consultation, yes/no & $<0.0001$ & 4.817 & 2.647 & 8.769 \\
\hline
\end{tabular}

\section{Discussion}

In selection of method for committing suicide, factors such as physical condition of individual, tendency to choose a death containing elements of either comfortable, calm or violent factors and seriousness of the death idea are important (7). On the other hand, easy availability and social acceptance of the method, familiarity to the related method by individual and related community are other determinants in selection of method for suicidal attempt. It is known that, chosen method for suicidal attempt is determined related to the available possibilities, but regional traditions are also suggested to play roles for this selection (8). The method of suicidal attempt is important in the aspect of desire for death. While it is not surprising for individuals who have a planned intention for suicide and strong desire for death to choose more lethal methods such as hanging, jumping from high places or cutting their wrists, the opposite statement is not true. The chosen method being non-aggressive may not mean that the intention to die is less. High dose drug ingestion may independently show the seriousness of death desire. However, most of times, suicidal attempts with such methods do not end fatally. Therefore, these kinds of suicidal attempts help individuals to achieve their goals in attracting the attention of others and expressing themselves, when they do not result with death. In a study conducted by Barr et al. the first row belonged to drug ingestion for the methods used by subjects who attempted suicide (9). While the rates of suicidal attempts with methods other than excessive drug ingestion were below $5 \%$ in the European countries (10), studies in our country revealed that in both genders, the most frequently preferred method for suicide was hanging (11). In the study by Demirci et al. conducted in the city of Konya, $81.6 \%$ of shotgun deaths were in males and $18.4 \%$ in females (12). In another study conducted by Asirdizer et al. in the city of İstanbul, 63\% of suicide-related deaths by shotgun were in males and 37\% in females (13). The ratio of aggressive methods for suicide was $12 \%$ in our study. As expected, the rate of aggressive suicide attempts was also higher in males, in our study. Since physical conditions and personality characteristics of males are different than females, they might have the tendency to prefer a suicide method that contains violence and anger. Since firearms have become easily accessible, the risk of suicide with firearms has been increased in the recent years. Shotgunrelated suicide has been increased significantly in the recent years due to their availability. It is a well-known fact that shotguns, which are easily available in rural areas, where they are usually used for security and in urban areas for hunting and sports, increase the risk of injury and death. New legal arrangements for possession and carrying shotguns and other firearms would reduce the number of suicides as well as other kinds of fatal incidents. While rates of aggressive suicide were similar in the village and city center ( $9.3 \%$ vs. $11.4 \%$ ), in district centers, this rate was $16.1 \%$, and there were no statistically significant 
differences between them. Studies performed on this topic showed that there were differences between rural and urban areas regarding the chosen method of suicide. While the rate of suicide by jumping from high places is increased in city centers, the most frequent method of suicide is asphyxia due to carbon monoxide exposure in peripheral regions in developed countries (8). In our study, suicidal attempt by carbon monoxide intoxication was identified only in four patients. In two patients, there were injuries related to cutting-piercing tools in addition to carbon monoxide poisoning. One reason for this may be that using carbon monoxide for suicide is not a traditional method in our region, being unrecognized or unknown. Another reason may be that admitted patients with intention to commit suicide by carbon monoxide poisoning may remain undercover or misleading physicians by telling them a false medical history. Admittance rates of males and females were similar in our study (75.7\% and $73.0 \%, \mathrm{P}=0.839$ ). However, for admittance to intensive care and surgical services, rates of males were significantly higher than females (ICU $26.6 \%$ vs. $4.8 \%$ and surgical services $26.6 \%$ vs. $16.9 \%, \mathrm{P}=0.047$ ). The reason may be that males have a higher preference rate for aggressive suicide attempts. Johnson et al. suggested that the reason for females to choose less lethal methods for suicide may be having more responsibility in family life; therefore, their roles in the family may play a protective role against successful suicidal attempts (14). When psychiatric characteristics were analyzed for chosen methods for suicide, the rate of consultation with psychiatry in patients with aggressive attempts is significantly less than those with non-aggressive attempts (32.8\% vs. $73.6 \%$, $\mathrm{P}<0.0001)$. This may be related to poor general condition of patients following aggressive attempts, making it impossible to consult with psychiatry. In our study, while non-aggressive suiciders were admitted to emergency service with a significantly higher rate than aggressive ones (76.5\% vs. 9.4\%, P<0.0001), aggressive suiciders were more frequently admitted to the surgical services (67.2\% vs. 13.6\%, $\mathrm{P}<0.0001)$. The reason may be having serious organ damages which need urgent care and operation in aggressive suiciders after jumping from high places, hanging or being injured by piercing-cutting tools. While aggressive suiciders were admitted urgently to the related service, non-aggressive suiciders were kept under observation for longer periods. Most patients admitted to the hospital for attempted suicide are treated and followed in emergency services and discharged uneventfully. The duration of their hospitalization shows differences among chosen methods for suicidal attempt. Patients who have previously attempted to commit suicide or having suicidal thoughts need specialized management in the emergency service. Therefore, during evaluation of patients by physicians working in the emergency service, a thorough medical history should be obtained to uncover the suicide attempt, the reason for committing suicide should be searched and tried to be solved, and to prevent recurring attempts for suicide, patients and their relatives should be directed to psychiatry clinics following the explanation of situation (7). In a study conducted in emergency services of England, traditional risk factors for parasuicide such as drug addiction, psychiatric follow-up, and previous history of self-poisoning were determined to be related at a higher level to psychosocial support or specialist follow-up. Another self-poisoning attempt was observed in approximately $15 \%$ of their patient group within six months. Furthermore, in the study, active follow-up of self-poisoning reduced the recurrence risk of parasuicide. In our study, we requested psychiatry consultation in $68.7 \%$ of our patients during their stay in the emergency service and tried to provide a specialist follow-up. According to our study, patients who committed aggressive suicide were consulted to psychiatry to a lesser extent in the emergency service (15). This might have been due to inability to provide psychiatric interview conditions secondary to poor general condition of patient or life-threatening physical injuries. Although, we believe that after patient acquired a satisfactory physical condition to interview, psychiatric consultation and follow-up could be requested by related specialist, we did not have relevant data on this subject in our study. In benign aggressive suicidal attempts such as minor incisions in the wrist, Patients refuse psychiatric consultation necessitating a waiting period of 3-4 hours in the emergency service. They also, leave the emergency service telling that they would later get help from psychiatric outpatient clinic. Regarding non-aggressive suicidal patients, since they are mostly poisoned by drugs, symptoms such as nausea, vomiting, abdominal pain, somnolence, headache, vertigo, agitation, urine retention or fatigue are more frequently found as expected. These patients are kept under observation for a long period and hospitalized in the emergency service and during this process, psychiatric consultation is also performed.

\subsection{Limitation}

This was a single-center study based on the expression of patients and patient relatives. Notifications could be wrong. Some cases may intent to hide their suicide attempt. A detailed psychiatric examination was not performed in emergency conditions. On the other hand, having a patient population of more than 500 and collecting data from surrounding 11 centers for nearly four years were the strong points of this study.

\section{Acknowledgements}

The authors would like to thank Ataturk University, Medical Faculty, Department of Emergency.

\section{Authors' Contributions}

Atif Bayramoglu: Designing the study, writing the manuscript. Sultan Tuna Akgol Gur: Patients examination. Murat Saritemur: Analyzing data. Mucahit Emet: Patients 
examination, statistical analysis. All authors read and approved the manuscript.

\section{References}

1. World Health Organization.. First WHO report on suicide prevention.: WHO; 2014. Available from: http://www.who.int/mediacentre/news/releases/2014/suicide-prevention-report/en/\#.

2. Ajdacic-Gross V, Weiss MG, Ring M, Hepp U, Bopp M, Gutzwiller $F$, et al. Methods of suicide: international suicide patterns derived from the WHO mortality database. Bull World Health Organ. 2008;86(9):726-32.

3. Emet M, Saritas A, Acemoglu H, Aslan S, Cakir Z. Predictors of missed injuries in hospitalized trauma patients in the emergency department. Eur J Trauma Emerg Surg. 2010;36(6):559-66.

4. Traskman L, Asberg M, Bertilsson L, Sjüstrand L. Monoamine metabolites in CSF and suicidal behavior. Arch Gen Psychiatry. 1981;38(6):631-6.

5. Schmidtke A, Bille-Brahe U, DeLeo D, Kerkhof A, Bjerke T, Crepet P, et al. Attempted suicide in Europe: rates, trends and sociodemographic characteristics of suicide attempters during the period 1989-1992. Results of the WHO/EURO Multicentre Study on Parasuicide. Acta Psychiatr Scand. 1996;93(5):327-38.

6. Ruesch S, Walder B, Tramer MR. Complications of central venous catheters: internal jugular versus subclavian access--a system- atic review. Crit Care Med. 2002;30(2):454-60.

7. Yanturalı, S. . Approach to patients with suicidal thoughts in the emergency department. J Emerg Med.2000:246-53.

8. Steele MM, Doey T. Suicidal Behaviour in Children and Ado lescents Part 1: Etiology and Risk Factors. Can J Psychiatry. 2007;52(6):21S-33s.

9. Barr W, Leitner M, Thomas J. Self-harm or attempted suicide? Do suicide notes help us decide the level of intent in those who survive? Accid Emerg Nurs. 2007;15(3):122-7.

10. Michel K, Ballinari P, Bille-Brahe U, Bjerke T, Crepet P, De Leo D, et al. Methods used for parasuicide: results of the WHO/EURO Multicentre Study on Parasuicide. Soc Psychiatry Psychiatr Epidemiol. 2000;35(4):156-63.

11. Altındag A, Sir A, Ozkan M. Changes in suicide rates in Turkey (1974-1998). Psychiatry Turk. 2001;2:79-86.

12. Demirci S, Dogan KH, Gunaydın G, Koc S. Deaths with shotguns. TurkJ. 2009;1(1):206-12.

13. Asirdizer M, Canturk G, Canturk N, Yavuz MS, Sari H. Analyses of suicidal deaths with shotguns in Istanbul, 1998-2007. Ulus Travma Acil Cerrahi Derg. 2010;16(1):47-53.

14. Johnson GR, Krug EG, Potter LB. Suicide among adolescents and young adults: a cross-national comparison of 34 countries. Suicide Life Threat Behav. 2000;30(1):74-82.

15. Kapur N, Cooper J, Hiroeh U, May C, Appleby L, House A. Emergency department management and outcome for self-poisoning: a cohort study. Gen Hosp Psychiatry. 2004;26(1):36-41. 